\title{
Social connection and exclusion of Australian women with no children during midlife
}

\author{
Beth Turnbull \\ Deakin University \\ Melissa L. Graham \\ Deakin University \\ Ann R. Taket \\ Deakin University
}

\begin{abstract}
Evidence indicates women with no children can experience pronatalism-driven stereotyping, stigmatisation and exclusion. This exploratory cross-sectional study described the social connection and exclusion of Australian women with no children during midlife (defined as aged 45 to 64 years). A total of 294 Australian midlife women with no children completed a self-administered online questionnaire. Data were collected on indicators of exclusion in the social, civic, service and economic domains of life, and participants' self-reported perceptions of being stereotyped, stigmatised and excluded because they have no children. Data were analysed using descriptive statistics and, for differences between involuntarily childless, circumstantially childless and voluntarily childless women: One Way ANOVAs for normally distributed continuous data, and Kruskal Wallis Analyses of Ranks and Chi Square Tests for Independence for categorical data and non-normally distributed continuous data. The findings indicate midlife women feel negatively stereotyped because they have no children. The extent and quality of midlife women's resources and participation vary between and within the domains of life. However, midlife women reported feeling more excluded because they have no children in the social and civic domains than the service and economic domains. There are few differences between typologies of women with no children. Given that social exclusion is a key social determinant of health and wellbeing, it is essential to ensure all women have opportunities for connection in all domains of life in Australian society regardless of their motherhood status, by challenging pronatalism at all levels of society.
\end{abstract}

Keywords: childlessness, voluntary, circumstantial, involuntary, social exclusion, stigma

Rising numbers of Australian women are having no children. In 2011, 13.6 per cent of Australian women between 45 and 64 years of age had no biological children, increasing from 12.5 per cent in 2006 (Australian Bureau of Statistics [ABS], 2007, 2012a).

Research suggests women with no children may experience social exclusion as a result of pronatalism, which constructs motherhood as central to being a woman, in Australian society (Rich, Taket, Graham, \& Shelley, 2011; Turnbull, Graham \& Taket, 2016; Veevers, 1979).

Corresponding author: Beth Turnbull (b.turnbull@deakin.edu.au) 
Social exclusion is defined as a multidimensional process driven by unequal power relationships interacting between the societal, community, relationship and individual levels; which is experienced as a continuum of connection and exclusion that limits the extent and quality of individuals' and groups' opportunities and resources for participating in the social, civic, service and economic domains of life (Levitas et al., 2007; Popay et al., 2008).

Pronatalism is one of a range of intersecting social constructs, such as gender, sexual orientation, race and age, which reflect hegemonic configurations of what is valued and not valued in society, and interact to produce diverse experiences of connection and exclusion (Butler, 2011; Collins, 2000; Popay et al., 2008). Pronatalism has particular salience for women (Veevers, 1979). At the societal-level, pronatalism takes the form of ideologies, discourses and policies that impose a moral, patriotic and economic imperative on women to reproduce (Graham \& Rich, 2012a; Veevers, 1979). Pronatalism thus reinforces patriarchal power by disempowering women as mothers with reduced resources; and stigmatising women with no children who do not conform to pronatalist norms (Mumtaz, Shahid, \& Levay, 2013). Analyses have revealed pronatalist discourses in Australian political and media rhetoric of motherhood as natural and patriotic, and of having no children as irresponsible, selfish, immature, hedonistic, discrediting and failing to contribute to society (Graham \& Rich, 2012a, 2012b; Heard, 2006; Sawer, 2013). These studies suggest pronatalism drives societal-level exclusionary processes which construct women with children as symbolically included 'insiders' and women with no children as deviant and excluded 'outsiders' (Carey et al., 2009). While it is important to acknowledge that pronatalism affects women with and without children in nuanced ways, this research focuses on women with no children, who are often invisible and overlooked in research (Graham, 2013).

There is evidence pronatalism pervades the community, relationship and individual levels through cultures, attitudes and beliefs of women with children as natural and inevitable, and women with no children as unnatural and discredited (Çopur \& Koropeckyj-Cox, 2010; Gillespie, 2000; Koropeckyj-Cox \& Pendell, 2007; LaMastro, 2001; Rich et al., 2011; Turnbull, Graham \& Taket, 2016). Research also suggests pronatalism-driven stigmatisation can vary between typologies of women with no children (Kopper \& Smith, 2001; Lampman \& Dowling-Guyer, 1995), including involuntarily childless women, who wanted to have biological children but could not achieve a viable pregnancy (Daniluk, 2001); circumstantially childless women, who could not have children as a result of circumstances such as partner infertility, having no partner, financial constraints, or health issues other than those preventing a viable pregnancy (Cannold, 2005); and voluntarily childless women, who freely chose not to have children (Veevers, 1979). However, there is little existing research investigating connection and exclusion in the social, civic, service and economic domains, for women with no children during midlife (aged 45 to 64 years). The social domain includes social networks, interaction with family and friends, social support, and participation in social and leisure activities (Albertini \& Mencarini, 2014; Levitas et al., 2007). The majority of studies in the social domain focus on women or adults with no children during later life (including age ranges 60 years and older) (see for example Cwikel, Gramotnev \& Lee, 2006; Dykstra \& Wagner, 2007; Grundy \& Read, 2012; Vikström et al., 2011; Wenger et al., 2007). A smaller body of research investigates women with no children in their reproductive years (see for example Albertini \& Mencarini, 2014; Koropeckyj-Cox, 2002; McNamee \& James, 2012; Turnbull, Graham \& Taket, 2016; Wagner, Wrzus, Neyer \& Lang, 2015). The extant research on the social networks, interaction, support and participation of women with no children is inconsistent and inconclusive; and provides no evidence on women during midlife. 
The civic domain includes volunteering and participating in community and political groups, activities and events (Levitas, 2006). The limited research in the civic domain investigates women with no children in their later years (Cwikel et al., 2006; Wenger et al., 2007) and reproductive years (Turnbull, Graham \& Taket, 2016). These studies are inconclusive, finding an increased likelihood of involvement in volunteering for unmarried Australian women with no children aged 73 to 78 years (Cwikel et al., 2006); no difference in participation in voluntary work or associations between 'late-life' adults with and without children from nine countries, including Australia (Wenger et al., 2007); and low levels of participation in community groups, activities and events by women with no children in their reproductive years (Turnbull, Graham \& Taket, 2016). There is no existing research in the civic domain on women with no children during midlife.

In the service domain, the extent to which services are available, accessible, affordable and appropriate needs to be considered (Levitas, 2006). There is limited and inconsistent research in the service domain on women with no children during their reproductive years and late life (Bernstein, 2001; Cwikel et al., 2006; Turnbull, Graham \& Taket, 2016) and no evidence relating to women with no children during midlife.

The economic domain includes financial and material resources, employment, and the quality of working lives (Levitas et al., 2007; Popay et al., 2008). The majority of research in the economic domain investigates women during their reproductive years (Abma \& Martinez, 2006; McNamee \& James, 2012; Miranti et al., 2009; Misra, Budig, \& Boeckmann, 2011; Turnbull, Graham \& Taket, 2016; Waren \& Pals, 2013), their working years (see for example Baumle, 2009; Berdahl \& Moon, 2013; Casper, Weltman, \& Kwesiga, 2007; Hamilton, Gordon, \& Whelan-Berry, 2006; Swanberg, Pitt-Catsouphes, \& Drescher-Burke, 2005), and later life (including age ranges above 65 years) (Cwikel et al., 2006; Dykstra \& Wagner, 2007; Hank \& Wagner, 2013; Koropeckyj-Cox \& Call, 2007). The limited research with women during midlife has found a positive association between having no children and higher incomes in the United States for married women aged 45 to 66 years (Huber, Bookstein, \& Fieder, 2010), and in Australia for women aged 40 to 54 years; but no independent association between having no children and higher occupational status in Australian women aged 40 to 54 years (Parr, 2005). The existing research provides little evidence that women with no children are at risk of exclusion from employment or material and financial resources. However, research suggests women with no children during their working years have low control over work hours (Swanberg et al., 2005); experience more harassment and mistreatment than other employees (Berdahl \& Moon, 2013); experience more job stress and less work-life balance than women with children (McNamee \& James, 2012); make less use of employment benefits than women with children (Hamilton et al., 2006); and perceive less equity in work opportunities, access to benefits, and respect for non-work roles than employees with children (Casper et al., 2007). There is, however, no research on the quality of working lives of women with no children during midlife.

As the foregoing discussion reveals, there is a dearth of research on the social connection and exclusion from multiple domains of life, of different typologies of midlife women with no children. Accordingly, this exploratory cross-sectional study aims to describe the social connection and exclusion in multiple domains of life of Australian women with no children aged 45 to 64 years, and asks: (1) what is the extent of social connection and exclusion of women with no children; and (2) does the extent of social connection and exclusion vary for different typologies of women with no children? 


\section{Method}

This exploratory, mixed-methods, cross-sectional study was conducted during 2015 in Australia. Deakin University's Human Ethics Advisory Group approved the research (HEAG-H 175-2013). This paper focuses on quantitative component of the study.

\section{Sampling and recruitment}

The target population was female Australian residents aged 45 to 64 years who were not currently pregnant, and had never assumed the role or identity of a biological or social mother (for example, of step, adopted or fostered-children). Due to the lack of a sampling frame of women with no children, a non-probability sample was recruited by promoting the study through newsletters and social media of 40 organisations, and six blogs and Facebook pages likely to be accessed by the target population. Of the 535 responses, 238 incomplete responses were excluded. In addition, three complete responses were excluded during screening as the respondents' ages fell outside the target age range. This resulted in a sample of 294 women, and a completion rate of 55 per cent.

\section{Data collection and measures}

Data on socio-demographics, typologies of women with no children, stigmatisation, stereotyping and exclusion were collected by way of a self-administered online questionnaire. Indicators of exclusion were measured using the social network, social interaction and subjective social support subscales of the Duke Social Support Index (George, Blazer, Hughes, \& Fowler, 1989), the MOS social support index (Chronbach's $\alpha=0.97$; one-year stability $\alpha=0.78$ ) (Sherbourne \& Stewart, 1991) and an adapted social and leisure participation scale (Melbourne Institute of Applied Economic and Social Research, 2012) in the social domain; participation in community groups, activities and events (Australian Bureau of Statistics, 2010), volunteering (Women's Health Australia, 2010), satisfaction with feeling part of the community (Cummins, Eckersley, Pallant, Van Vugt, \& Misajon, 2003) and satisfaction with opportunities to have a say on important issues (Pope \& Zhang, 2010 ) in the civic domain; degree of problems accessing and using services (Dermott et al., 2012) in the service domain; and employment status (Melbourne Institute of Applied Economic and Social Research, 2012), personal income (Australian Bureau of Statistics, 2011) and work-life conflict (Waumsley, Houston, \& Marks, 2010) in the economic domain.

Participants' self-reported perceptions of being stereotyped and stigmatised by others because they have no children were measured using adapted stigma consciousness (Pinel, 1999) and stereotype perception (Somers, 1993) scales. Fivepoint scales measured participants' self-reported degree of feeling excluded from domains of life due to having no children by asking participants, 'To what degree have you felt excluded from the following areas of life because you have no children?': support provided by family and friends; contact with family and friends; participation in social and leisure activities; participation in community life; accessing and using services; employment; and material and financial resources.

\section{Data analysis}

Aggregate scores were computed for social networks, social interaction, social support, subjective social support, social and leisure participation, community participation, problems accessing and using services, work-life conflict, stigma consciousness and perceived stereotyping (Hughes, Blazer, \& Hybels, 1990; Pinel, 
1999; Somers, 1993; Waumsley et al., 2010). Items on paid work status and unemployment were used to compute employment status. Personal income ranges were recoded into imputed income (ABS 2012b) then income levels (ABS 2013). Five-point scales measuring degree of perceived exclusion due to being childless were recoded into 'no degree of perceived exclusion' and 'at least a slight degree of perceived exclusion'.

Descriptive statistics were used to describe socio-demographics, indicators of exclusion, and perceived stigmatisation, stereotyping and exclusion due to having no children. Parametric (one way ANOVAs and Scheffe's post-hoc) tests analysed differences between types of childless women in relation to normally distributed continuous variables, including social and leisure participation, work-life conflict, stigma consciousness and perceived stereotyping. Non-parametric (Kruskal Wallis $\mathrm{H}$ and Dunn's post-hoc) tests analysed differences in categorical variables or nonnormally distributed continuous variables, including social networks, social interaction, social support, subjective social support, community participation, satisfaction with feeling part of the community, satisfaction with opportunities to have a say, problems accessing or using services, employment status, personal income and the self-reported degree to which participants felt excluded from domains of life because they had no children. The non-parametric Chi Square Test for Independence analysed differences in volunteering participation, a binary categorical variable. A conservative significance level of 0.01 was used for reporting results as statistically significant.

\section{Results}

\section{Demographic characteristics and typologies of women with no children}

Table 1 shows the demographic characteristics of participants as well as comparable Australian data. A greater percentage of participants $(69.4 \% ; n=204)$ than Australian females (54.3\%) (Australian Bureau of Statistics, 2012a) was aged between 45 and 54 years. A slightly lower percentage of participants $(64.5 \%$; $n=189)$ was in a relationship than the percentage of Australian females aged 45 to 64 years in a registered or de facto marriage $(70.3 \%)$ (Australian Bureau of Statistics, 2012a). A higher percentage of participants identified as lesbian or bisexual $(7.8 \% ; n=12)$ than Australian women aged 16 to 59 years (2.2\%) (Smith et al., 2003). A substantially greater percentage of participants $(76.2 \% ; n=224)$ than Australian women over 15 years of age (19.9\%) possessed a bachelor degree or higher (Australian Bureau of Statistics, 2012a). A higher percentage of participants (81.9\%; $n=241)$ than Australian women over the age of $15(71.6 \%)$ was employed, while a lower percentage of participants $(6.1 \% ; n=18)$ than Australian women aged over 15 years $(24.5 \%)$ was not in the labour force (Australian Bureau of Statistics, 2012a). Over half $(55.7 \% ; n=151)$ the participants were in the high-income bracket, compared to 20 per cent of Australians (Australian Bureau of Statistics, 2013). 
Table 1. Demographic characteristics of participants and comparable Australian data

\begin{tabular}{|c|c|c|c|}
\hline & $\begin{array}{l}\text { Participants } \\
\text { per cent }(n)\end{array}$ & $\begin{array}{l}\text { Australian } \\
\text { per cent }\end{array}$ & $\begin{array}{c}\text { Percentage } \\
\text { difference (95\% } \\
\mathrm{Cl}) \\
\end{array}$ \\
\hline \multicolumn{4}{|l|}{ Age $(n=294)$} \\
\hline $45-49$ & $36.7(108)$ & 27.6 & $\begin{array}{c}9.1(3.83- \\
14.78)\end{array}$ \\
\hline $50-54$ & $32.7(96)$ & 26.7 & $6.0(0.85-11.5)$ \\
\hline $55-59$ & $22.8(67)$ & 23.8 & $-1.0(-5.4-4.1)$ \\
\hline $60-64$ & $7.8(23)$ & $21.9^{1}$ & $\begin{array}{c}-14.1(-16.6-- \\
10.4)\end{array}$ \\
\hline \multicolumn{4}{|l|}{ Relationship status $(n=293)$} \\
\hline Single & $35.5(104)$ & 29.7 & $5.8(0.5-11.6)$ \\
\hline Partnered & $64.5(189)$ & $70.3^{2}$ & $\begin{array}{c}-5.8(-11.4-- \\
0.54)\end{array}$ \\
\hline \multicolumn{4}{|l|}{ Sexual orientation $(n=154)$} \\
\hline Lesbian or bisexual & $7.8(12)$ & $2.2^{3}$ & $5.6(2.3-10.9)$ \\
\hline Heterosexual & $92.2(142)$ & 97.8 & $\begin{array}{c}-5.6(-10.9-- \\
2.3)\end{array}$ \\
\hline \multicolumn{4}{|l|}{ Educational attainment $(n=294)$} \\
\hline Year 12 or below & $9.2(27)$ & 58.6 & $\begin{array}{c}-49.4(-52.2-- \\
45.6)\end{array}$ \\
\hline Certificate/diploma & $14.6(43)$ & 21.5 & $\begin{array}{c}-6.9(-10.5-- \\
2.4)\end{array}$ \\
\hline Bachelor degree & $25.2(74)$ & 14.8 & $10.4(5.8-15.6)$ \\
\hline $\begin{array}{l}\text { Graduate/postgraduate } \\
\text { qualification }\end{array}$ & $51.0(150)$ & $5.1^{4}$ & $\begin{array}{c}45.9(40.2- \\
51.6)\end{array}$ \\
\hline \multicolumn{4}{|l|}{ Employment status $(n=294)$} \\
\hline Employed/self employed & $81.9(241)$ & 71.6 & $10.3(5.6-14.4)$ \\
\hline Unemployed & $11.9(35)$ & 3.9 & $8.0(4.8-12.2)$ \\
\hline Not in labour force & $6.1(18)$ & $24.5^{5}$ & $\begin{array}{c}-18.4(-20.6-- \\
15.0)\end{array}$ \\
\hline \multicolumn{4}{|l|}{ Personal income level $(n=271)$} \\
\hline Low (first three deciles) & $11.4(31)$ & 30.0 & $\begin{array}{c}-18.6(-21.8-- \\
14.2)\end{array}$ \\
\hline Lower middle (fourth decile) & $6.6(18)$ & 10.0 & $-3.4(-5.8-0.25)$ \\
\hline Middle (third quintile) & $10.3(28)$ & 20.0 & $\begin{array}{c}-9.7(-12.8-- \\
5.5)\end{array}$ \\
\hline Upper middle (fourth quintile) & $15.9(43)$ & 20.0 & $-4.1(-8.0-0.7)$ \\
\hline High (fifth quintile) & $55.7(151)$ & $20.0^{5}$ & $\begin{array}{c}35.7(29.8- \\
41.5)\end{array}$ \\
\hline \multicolumn{4}{|l|}{ State of residence $(n=294)$} \\
\hline Australian Capital Territory & $6.1(18)$ & 1.7 & $4.4(2.2-7.7)$ \\
\hline New South Wales & $26.9(79)$ & 32.2 & $\begin{array}{c}-5.3(-10.1- \\
0.01)\end{array}$ \\
\hline Northern Territory & $3.7(11)$ & 0.9 & $2.8(2.2-5.6)$ \\
\hline Queensland & $13.6(40)$ & 20.1 & $-6.5(-10.0--2.1$ \\
\hline South Australia & $3.4(10)$ & 7.4 & $-4.0(-5.6--1.3)$ \\
\hline Tasmania & $4.4(13)$ & 2.3 & $2.1(0.3-5.1)$ \\
\hline Victoria & $31.0(91)$ & 25.0 & $6.0(0.9-11.5)$ \\
\hline Western Australia & $10.9(32)$ & $10.2^{6}$ & $0.7(-9.4-4.7)$ \\
\hline \multicolumn{4}{|l|}{ Geographical residence $(n=293)$} \\
\hline Major city & $60.8(178)$ & 68.4 & $\begin{array}{l}-7.6(-13.3-- \\
2.2)\end{array}$ \\
\hline Inner regional & $21.5(63)$ & 19.7 & $1.8(-2.5-6.9)$ \\
\hline
\end{tabular}




\begin{tabular}{lccc} 
Outer regional & $14.0(41)$ & 9.5 & $4.5(1.0-8.9)$ \\
Remote & $3.4(10)$ & 1.5 & $1.9(0.4-4.7)$ \\
Very remote & $0.3(1)$ & $0.8^{7}$ & $-0.5(-0.7-1.1)$ \\
\hline
\end{tabular}

Notes: 1 Australian females aged 45 - 64 (Australian Bureau of Statistics, 2012a); 2 Australian females aged $45-64$ in registered or de facto marriages, or not married (Australian Bureau of Statistics, 2012a); 3 Australian females aged 16 to 59 years (Smith et al., 2003); 4 Australian females aged 15 years and over (Australian Bureau of Statistics, 2012a); 5 Income deciles of Australian adults (Australian Bureau of Statistics, 2013); 6 Australian females (Australian Bureau of Statistics, 2012a); 7 Australian residents (Australian Bureau of Statistics, 2008).

Table 2 shows socio-demographic differences between typologies of women with no children. Voluntarily childless women constituted the largest group $(53.3 \% ; n=155)$, followed by circumstantially childless women $(35.1 \% ; n=102)$, involuntarily childless women $(8.9 \% ; n=26)$, and women who had no children for unspecified or other reasons, including having no living children $(3.7 \% ; n=11)$. Circumstantially childless women were more likely to have no partner than involuntarily and voluntarily childless women $(X=9.9 ; \mathrm{df}=2 ; p=0.007)$.

Table 2. Differences in demographic characteristics of typologies of women with no children $^{1}$

\begin{tabular}{|c|c|c|c|c|c|c|}
\hline & Involuntary & Circumstantial & Voluntary & Statistic & df & p value \\
\hline $\begin{array}{l}\text { Age in years }(n= \\
283)\end{array}$ & $n=26$ & $n=102$ & $\mathrm{n}=155$ & & & \\
\hline Mean & $\begin{array}{c}51.3(\mathrm{SD}= \\
5.2)\end{array}$ & $51.6(\mathrm{SD}=4.5)$ & $\begin{array}{c}52.0 \\
(S D=5.2)\end{array}$ & $0.3^{2}$ & 2 & 0.76 \\
\hline $\begin{array}{l}\text { Relationship status } \\
(\mathrm{n}=282)\end{array}$ & $\mathrm{n}=26$ & $\mathrm{n}=101$ & $\mathrm{n}=155$ & & & \\
\hline Single & $5.9 \%$ & $47.5 \%$ & $46.5 \%$ & $9.9^{3}$ & 2 & 0.007 \\
\hline Partnered & $11.0 \%$ & $29.3 \%$ & $59.7 \%$ & & & \\
\hline $\begin{array}{l}\text { Sexual orientation } \\
(n=149)\end{array}$ & $n=19$ & $\mathrm{n}=81$ & $n=49$ & & & \\
\hline $\begin{array}{l}\text { Lesbian or } \\
\text { bisexual }\end{array}$ & $9.1 \%$ & $54.5 \%$ & $36.4 \%$ & $N / A^{4}$ & & \\
\hline Heterosexual & $13.0 \%$ & $54.3 \%$ & $32.6 \%$ & & & \\
\hline $\begin{array}{l}\text { Geographic } \\
\text { residence }(n=283)\end{array}$ & $n=26$ & $n=102$ & $n=155$ & & & \\
\hline Major city & $9.9 \%$ & $34.5 \%$ & $55.6 \%$ & $0.6^{3}$ & 2 & 0.74 \\
\hline Regional/remote & $8.0 \%$ & $38.4 \%$ & $53.6 \%$ & & & \\
\hline Education $(n=283)$ & $\mathrm{n}=26$ & $n=102$ & $n=155$ & & & \\
\hline Year 11 or below & $15.4 \%$ & $7.8 \%$ & $4.5 \%$ & $2.8^{5}$ & 2 & 0.25 \\
\hline $\begin{array}{l}\text { Year } 12 \text { or } \\
\text { equivalent }\end{array}$ & $7.7 \%$ & $0.0 \%$ & $3.2 \%$ & & & \\
\hline $\begin{array}{l}\text { Certificate or } \\
\text { diploma }\end{array}$ & $11.5 \%$ & $13.7 \%$ & $14.8 \%$ & & & \\
\hline $\begin{array}{l}\text { Bachelor or } \\
\text { above }\end{array}$ & $65.4 \%$ & $78.4 \%$ & $77.4 \%$ & & & \\
\hline Mean rank & 123.1 & 144.4 & 143.6 & & & \\
\hline
\end{tabular}

Notes: 1 Excluding 'other' women and women who did not specify type of childlessness; 2 One Way ANOVA; 3 Chi Square Test for Independence; 4 Assumptions of Chi Square Test for Independence not met due to cells with count less than five; 5 Kruskal Wallis Analysis of Ranks.

Indicators of exclusion in the social, civic, service and economic domains

Table 3 shows the results for social networks, social interaction, social support, and social and leisure participation as indicators of the extent of resources and participation; and subjective social support as an indicator of the quality of resources. Circumstantially childless women reported lower levels of social support than 
voluntarily childless women (Dunn's Test $=-40.0 ; p<0.001$ ). Similarly, in a difference that approached significance at the 0.01 level, circumstantially childless women had lower subjective social support scores, suggesting they had lower levels of satisfaction with social support, than voluntarily childless women (Dunn's test = 25.7; $p=0.02$ ).

Table 3. Indicators of exclusion in the social domain

\begin{tabular}{|c|c|c|c|c|c|c|c|}
\hline & $\begin{array}{c}\text { All } \\
\text { women }\end{array}$ & Involuntary & Circumstantial & Voluntary & Statistic & df & $\begin{array}{c}p \\
\text { value }\end{array}$ \\
\hline $\begin{array}{l}\text { Social } \\
\text { networks }^{3}\end{array}$ & $\mathrm{n}=271$ & $n=26$ & $n=102$ & $n=155$ & & & \\
\hline Median & 3 & 3 & 3 & 3 & & & \\
\hline $\begin{array}{l}\text { Mean } \\
\text { rank }\end{array}$ & & 130.1 & 129.1 & 134.3 & $0.3^{1}$ & 2 & 0.86 \\
\hline $\begin{array}{l}\text { Social } \\
\text { interaction }\end{array}$ & $n=286$ & $n=25$ & $\mathrm{n}=98$ & $n=152$ & & & \\
\hline Median & 7 & 7 & 7 & 7 & & & \\
\hline $\begin{array}{l}\text { Mean } \\
\text { rank }\end{array}$ & & 137.3 & 139.4 & 137.2 & $0.05^{1}$ & 2 & 0.98 \\
\hline $\begin{array}{l}\text { Social } \\
\text { support }^{5}\end{array}$ & $n=271$ & $n=24$ & $n=93$ & $n=145$ & & & \\
\hline Mean & $\begin{array}{l}51.9 \\
(S D= \\
20.4)\end{array}$ & $\begin{array}{c}54.9 \\
(\mathrm{SD}=17.6)\end{array}$ & $\begin{array}{c}45.4 \\
(S D=20.6)\end{array}$ & $\begin{array}{c}55.8 \\
(S D= \\
19.6)\end{array}$ & & & \\
\hline $\begin{array}{l}\text { Mean } \\
\text { rank }\end{array}$ & & 139.7 & 106.3 & 146.73 & $16.2^{1}$ & 2 & $\begin{array}{c}<0.00 \\
1\end{array}$ \\
\hline $\begin{array}{l}\text { Social and } \\
\text { leisure } \\
\text { participation } \\
6\end{array}$ & $n=294$ & $n=26$ & $n=102$ & $n=155$ & & & \\
\hline Mean & $\begin{array}{c}43.7 \\
(S D= \\
10.9)\end{array}$ & $\begin{array}{c}41.5 \\
(\mathrm{SD}=9.6)\end{array}$ & $\begin{array}{c}42.6 \\
(\mathrm{SD}=11.4)\end{array}$ & $\begin{array}{c}44.5 \\
(S D= \\
10.8)\end{array}$ & $1.4^{2}$ & 2 & 0.25 \\
\hline $\begin{array}{l}\text { Subjective } \\
\text { social } \\
\text { support }^{7}\end{array}$ & $\mathrm{n}=260$ & $n=22$ & $\mathrm{n}=96$ & $n=133$ & & & \\
\hline $\begin{array}{l}\text { Median } \\
\text { Mean } \\
\text { rank }\end{array}$ & 25.5 & $\begin{array}{c}25.5 \\
112.5\end{array}$ & $\begin{array}{c}25 \\
112.3\end{array}$ & $\begin{array}{c}26 \\
138.1\end{array}$ & $7.97^{1}$ & 2 & 0.019 \\
\hline \multicolumn{8}{|c|}{$\begin{array}{l}\text { Notes: } 1 \text { Kruskal Wallis Analysis of Ranks; } 2 \text { One Way ANOVA; } 3 \text { Ordinal scale from } 0 \text { to } 17 \text {, with higher } \\
\text { scores indicating larger social network sizes; } 4 \text { Ordinal scale from } 0 \text { to } 13 \text {, with higher scores indicating } \\
\text { more social interaction; } 5 \text { Interval scale from } 0 \text { to } 76, \text { with higher scores indicating greater levels of } \\
\text { instrumental and affective social support (scores not normally distributed); } 6 \text { Interval scale from } 0 \text { to } 108 \text {, } \\
\text { with higher scores indicating greater participation in social and leisure activities; } 7 \text { Ordinal scale from } 10 \\
\text { to 29, with higher scores indicating greater subjective social support. }\end{array}$} \\
\hline \multicolumn{8}{|c|}{$\begin{array}{l}\text { Table } 4 \text { shows the results in the civic domain for community participation and } \\
\text { volunteering as indicators of the extent of participation; and satisfaction with feeling } \\
\text { part of the community and opportunities to have a say as indicators of the quality of } \\
\text { participation. Table } 4 \text { also shows the results in the service domain for degree of } \\
\text { problems accessing and using services as an indicator of the quality of participation; } \\
\text { and in the economic domain for employment status and personal income levels as } \\
\text { indicators of the extent of resources and participation, and work-life conflict as an } \\
\text { indicator of the quality of participation. }\end{array}$} \\
\hline
\end{tabular}


There were no differences between typologies of women with no children in the civic and economic domains. In the service domain, circumstantially childless women reported a greater degree of problems accessing and using services than voluntarily childless women (Dunn's test $=-28.3 ; p=0.01$ ).

Table 4. Indicators of exclusion in the civic, service and economic domains

\begin{tabular}{|c|c|c|c|c|c|c|c|}
\hline & $\begin{array}{c}\text { All } \\
\text { women }\end{array}$ & Involuntary & Circumstantial & Voluntary & Statistic & $\begin{array}{l}d \\
f\end{array}$ & $\begin{array}{c}\mathbf{p} \\
\text { value }\end{array}$ \\
\hline $\begin{array}{l}\text { Community } \\
\text { groups/ } \\
\text { events/activiti } \\
\text { es } \\
\text { participated } \\
\text { in }{ }^{4}\end{array}$ & $n=294$ & $n=26$ & $n=102$ & $\mathrm{n}=155$ & & & \\
\hline Mean & $\begin{array}{c}4.0 \\
(S D=2.5)\end{array}$ & $\begin{array}{c}3.4 \\
(S D=2.3)\end{array}$ & $\begin{array}{c}4.3 \\
(\mathrm{SD}=2.6)\end{array}$ & $\begin{array}{c}3.8 \\
(S D=2.4)\end{array}$ & & & \\
\hline Mean rank & & 120.7 & 152.7 & 138.5 & $3.9^{1}$ & 2 & 0.15 \\
\hline $\begin{array}{l}\text { Participation } \\
\text { in } \\
\text { volunteering }\end{array}$ & $n=294$ & $n=26$ & $n=102$ & $n=155$ & & & \\
\hline $\begin{array}{l}\text { Volunteer } \\
\mathrm{s}\end{array}$ & $51.4 \%$ & $10.3 \%$ & $34.6 \%$ & $55.1 \%$ & $0.5^{2}$ & 2 & 0.8 \\
\hline $\begin{array}{l}\text { Does not } \\
\text { volunteer }\end{array}$ & $48.6 \%$ & $8.2 \%$ & $37.4 \%$ & $54.4 \%$ & & & \\
\hline $\begin{array}{l}\text { Satisfaction } \\
\text { with feeling } \\
\text { part of the } \\
\text { community }\end{array}$ & $n=293$ & $n=26$ & $\mathrm{n}=101$ & $\mathrm{n}=155$ & & & \\
\hline $\begin{array}{l}\text { Median } \\
\text { Mean rank }\end{array}$ & 5.0 & $\begin{array}{c}5.0 \\
129.1\end{array}$ & $\begin{array}{c}5.0 \\
131.5\end{array}$ & $\begin{array}{c}6.0 \\
151.1\end{array}$ & $4.5^{1}$ & 2 & 0.1 \\
\hline $\begin{array}{l}\text { Satisfaction } \\
\text { with } \\
\text { opportunities } \\
\text { to have a say } \\
\text { on important } \\
\text { issues }^{5}\end{array}$ & $n=294$ & $n=26$ & $n=102$ & $n=155$ & & & \\
\hline $\begin{array}{l}\text { Median } \\
\text { Mean rank }\end{array}$ & 6.0 & $\begin{array}{c}5.0 \\
126.5\end{array}$ & $\begin{array}{c}5.0 \\
131.0\end{array}$ & $\begin{array}{c}6.0 \\
150.8\end{array}$ & $4.9^{1}$ & 2 & 0.09 \\
\hline $\begin{array}{l}\text { Degree of } \\
\text { problems } \\
\text { accessing or } \\
\text { using } \\
\text { services }^{6}\end{array}$ & $n=294$ & $n=26$ & $\mathrm{n}=102$ & $\mathrm{n}=155$ & & & \\
\hline Mean & $\begin{array}{c}71.8 \\
(\mathrm{SD}=5.6)\end{array}$ & $\begin{array}{c}72.2 \\
(\mathrm{SD}=4.5)\end{array}$ & $\begin{array}{c}70.8 \\
(\mathrm{SD}=6.6)\end{array}$ & $\begin{array}{c}72.0 \\
(\mathrm{SD}=4.2)\end{array}$ & & & \\
\hline $\begin{array}{l}\quad \text { Mean rank } \\
\text { Employment } \\
\text { status }\end{array}$ & $\mathrm{n}=294$ & $\begin{array}{c}138.6 \\
n=26\end{array}$ & $\begin{array}{c}125.3 \\
n=102\end{array}$ & $\begin{array}{c}153.6 \\
n=155\end{array}$ & $8.5^{1}$ & 2 & 0.01 \\
\hline $\begin{array}{l}\text { Employed/ } \\
\text { self- } \\
\text { employed }\end{array}$ & $72.4 \%$ & $76.9 \%$ & $70.6 \%$ & $73.5 \%$ & & & \\
\hline $\begin{array}{l}\text { Underemp } \\
\text { loyed }\end{array}$ & $9.5 \%$ & $15.4 \%$ & $7.8 \%$ & $9.0 \%$ & & & \\
\hline $\begin{array}{l}\text { Unemploy } \\
\text { ed }\end{array}$ & $11.9 \%$ & $3.8 \%$ & $13.7 \%$ & $12.3 \%$ & & & \\
\hline
\end{tabular}




\begin{tabular}{|c|c|c|c|c|c|c|c|}
\hline $\begin{array}{l}\text { Not in } \\
\text { labour } \\
\text { force }\end{array}$ & $6.1 \%$ & $3.8 \%$ & $7.8 \%$ & $5.2 \%$ & & & \\
\hline Mean rank & & 151.1 & 138.3 & 142.9 & $0.91^{1}$ & 2 & 0.63 \\
\hline Personal & & & & & & & \\
\hline $\begin{array}{l}\text { income } \\
\text { levels }\end{array}$ & $n=271$ & $\mathrm{n}=25$ & $\mathrm{n}=96$ & $n=262$ & & & \\
\hline Low & $11.4 \%$ & $4.0 \%$ & $12.5 \%$ & $11.3 \%$ & & & \\
\hline $\begin{array}{l}\text { Lower } \\
\text { middle }\end{array}$ & $6.6 \%$ & $16.0 \%$ & $7.3 \%$ & $5.0 \%$ & & & \\
\hline Middle & $10.3 \%$ & $12.0 \%$ & $9.4 \%$ & $11.3 \%$ & & & \\
\hline $\begin{array}{l}\text { Upper } \\
\text { middle }\end{array}$ & $15.9 \%$ & $20.0 \%$ & $15.6 \%$ & $14.9 \%$ & & & \\
\hline High & $55.7 \%$ & $48.0 \%$ & $55.2 \%$ & $57.4 \%$ & & & \\
\hline Mean rank & & 124.3 & 130.1 & 133.8 & $0.46^{1}$ & 2 & 0.79 \\
\hline $\begin{array}{l}\text { Work-life } \\
\text { conflict }^{7}\end{array}$ & $n=239$ & $n=23$ & $\mathrm{n}=80$ & $\mathrm{n}=127$ & & & \\
\hline Mean & $\begin{array}{c}18.3 \\
(\mathrm{SD}=8.5)\end{array}$ & $\begin{array}{c}17.9 \\
(\mathrm{SD}=7.5)\end{array}$ & $\begin{array}{c}17.9 \\
(\mathrm{SD}=8.1)\end{array}$ & $\begin{array}{c}18.9 \\
(\mathrm{SD}=9.0)\end{array}$ & $0.38^{3}$ & 2 & 0.68 \\
\hline
\end{tabular}

\section{Perceived stigmatisation, stereotyping and exclusion due to having no children}

Table 5 shows results for participants' self-reported perceptions of feeling stereotyped, stigmatised and excluded from different domains of life because they have no children. There were no differences in perceived stereotyping scores $(F=$ $0.3 ; \mathrm{df}=2 ; \mathrm{p}=0.7)$, or stigma consciousness scores $(\mathrm{F}=3.1 ; \mathrm{df}=2 ; \mathrm{p}=0.045)$, although the latter approached significance at the 0.01 level.

Of all participants, at least a slight degree of feeling excluded because they have no children was reported in the social domain by 46.9 per cent $(n=137)$ from contact with family and friends, 46.8 per cent $(n=137)$ from support provided by family and friends, and 46.8 per cent $(n=137)$ from participating in social and leisure activities; in the civic domain by 39.9 per cent $(n=117)$ from participating in community life; in the service domain by 18.4 per cent $(n=54)$ from accessing and using services; and in the economic domain by 18.8 per cent $(n=55)$ from material and financial resources, and 15.1 per cent $(n=44)$ from employment. Some differences in the social domain approached significance at the 0.01 level, with involuntarily childless women more likely than voluntarily childless women to report feeling excluded because they have no children from contact with family and friends (Dunn's test = 37.3; $p=0.05$ ) and support provided by family and friends (Dunn's test $=-39.5 ; p=$ $0.04)$. 
Table 5. Perceived stereotyping, stigmatisation and exclusion due to being childless

\begin{tabular}{|c|c|c|c|c|c|c|c|}
\hline & $\begin{array}{c}\text { All } \\
\text { women } \\
(n= \\
\left.282^{3}\right)\end{array}$ & $\begin{array}{c}\text { Involuntary } \\
\left(n=26^{4}\right)\end{array}$ & $\begin{array}{l}\text { Circumstantial } \\
\quad\left(n=102^{5}\right)\end{array}$ & $\begin{array}{l}\text { Voluntary } \\
\left(n=154^{6}\right)\end{array}$ & Statistic & $\begin{array}{l}d \\
f\end{array}$ & $\begin{array}{c}p \\
\text { value }\end{array}$ \\
\hline \multicolumn{8}{|l|}{$\begin{array}{l}\text { Stereotype } \\
\text { perception }^{7}\end{array}$} \\
\hline Mean & $\begin{array}{l}47.3 \\
(\mathrm{SD}= \\
14.7)\end{array}$ & $\begin{array}{c}45.7 \\
(S D=13.4)\end{array}$ & $\begin{array}{c}48.1 \\
(S D=13.0)\end{array}$ & $\begin{array}{c}47.0 \\
(S D=16.0)\end{array}$ & $0.3^{1}$ & 2 & 0.7 \\
\hline \multicolumn{8}{|l|}{$\begin{array}{l}\text { Stigma } \\
\text { consciousness } \\
8\end{array}$} \\
\hline Mean & $\begin{array}{c}41.9 \\
(S D= \\
6.4)\end{array}$ & $\begin{array}{c}40.3 \\
(\mathrm{SD}=7.1)\end{array}$ & $\begin{array}{c}41.0 \\
(S D=6.0)\end{array}$ & $\begin{array}{c}42.8 \\
(S D=6.4)\end{array}$ & $3.1^{1}$ & 2 & 0.045 \\
\hline \multicolumn{8}{|l|}{$\begin{array}{l}\text { Exclusion } \\
\text { social contact }^{9}\end{array}$} \\
\hline $\begin{array}{l}\text { Median } \\
\text { Mean rank }\end{array}$ & 5.0 & $\begin{array}{c}4.0 \\
112.1\end{array}$ & $\begin{array}{c}4.5 \\
135.7\end{array}$ & $\begin{array}{c}5.0 \\
149.4\end{array}$ & $6.5^{2}$ & 2 & 0.04 \\
\hline \multicolumn{8}{|l|}{$\begin{array}{l}\text { Exclusion } \\
\text { social support }\end{array}$} \\
\hline $\begin{array}{l}\text { Median } \\
\text { Mean rank }\end{array}$ & 5.0 & $\begin{array}{c}4.0 \\
110.7\end{array}$ & $\begin{array}{c}4.0 \\
136.2\end{array}$ & $\begin{array}{c}5.0 \\
150.2\end{array}$ & $7.1^{2}$ & 2 & 0.03 \\
\hline \multicolumn{8}{|l|}{$\begin{array}{l}\text { social/leisure } \\
\text { participation }^{9}\end{array}$} \\
\hline $\begin{array}{l}\text { Median } \\
\text { Mean rank }\end{array}$ & 5.0 & $\begin{array}{c}4.0 \\
115.4\end{array}$ & $\begin{array}{c}4.0 \\
135.5\end{array}$ & $\begin{array}{c}5.0 \\
149.9\end{array}$ & $5.9^{2}$ & 2 & 0.054 \\
\hline \multicolumn{8}{|l|}{$\begin{array}{l}\text { Exclusion } \\
\text { community life }\end{array}$} \\
\hline $\begin{array}{l}\text { Median } \\
\text { Mean rank }\end{array}$ & 5.0 & $\begin{array}{c}5.0 \\
137.1\end{array}$ & $\begin{array}{c}5.0 \\
129.1\end{array}$ & $\begin{array}{c}5.0 \\
150.4\end{array}$ & $5.6^{2}$ & 2 & 0.06 \\
\hline \multicolumn{8}{|l|}{$\begin{array}{l}\text { Exclusion } \\
\text { services }^{9}\end{array}$} \\
\hline $\begin{array}{l}\text { Median } \\
\text { Mean rank }\end{array}$ & 5.0 & $\begin{array}{c}5.0 \\
123.2\end{array}$ & $\begin{array}{c}5.0 \\
141.2\end{array}$ & $\begin{array}{c}5.0 \\
144.8\end{array}$ & $3.6^{2}$ & 2 & 0.17 \\
\hline \multicolumn{8}{|l|}{$\begin{array}{l}\text { Exclusion } \\
\text { employment }{ }^{9}\end{array}$} \\
\hline $\begin{array}{l}\text { Median } \\
\text { Mean rank }\end{array}$ & 5.0 & $\begin{array}{c}5.0 \\
134.0\end{array}$ & $\begin{array}{c}5.0 \\
141.3\end{array}$ & $\begin{array}{c}5.0 \\
142.9\end{array}$ & $0.7^{2}$ & 2 & 0.7 \\
\hline \multicolumn{8}{|l|}{$\begin{array}{l}\text { Exclusion } \\
\text { material/financi } \\
\text { al resources }\end{array}$} \\
\hline $\begin{array}{l}\text { Median } \\
\text { Mean rank }\end{array}$ & 5.0 & $\begin{array}{c}5.0 \\
128.4\end{array}$ & $\begin{array}{c}5.0 \\
139.7\end{array}$ & $\begin{array}{c}5.0 \\
144.9\end{array}$ & $2.2^{2}$ & 2 & 0.3 \\
\hline $\begin{array}{l}\text { Notes: } 1 \\
269 \text { for s } \\
\text { stereotyp } \\
\text { consciou } \\
\text { from soc } \\
\text { perceive } \\
70, \text { with } \\
\text { to } 5 \text {, with } \\
\text { they had }\end{array}$ & $\begin{array}{l}\text { One Way Al } \\
\text { igma consci } \\
\text { e perceptior } \\
\text { sness; } 6 n= \\
\text { al interactiol } \\
\text { Inegative st } \\
\text { cores belon } \\
\text { scores of } 4 \\
\text { no children. }\end{array}$ & $\begin{array}{l}\text { OVA; } 2 \text { Kruskal } \\
\text { usness, } 281 \text { for } \\
25 \text { for stigma co } \\
143 \text { for stereotyp } \\
\text { and education; } 7 \\
\text { reotyping by oth } \\
40 \text { indicating stig } \\
\text { r below indicatin }\end{array}$ & $\begin{array}{l}\text { allis Analysis of Rank } \\
\text { xclusion from social in } \\
\text { sciousness; } 5 n=95 \\
\text { perception, } 151 \text { for st } \\
\text { Aggregate index from } \\
\text { rs because they have } \\
\text { ha consciousness due } \\
\text { participants felt at lea }\end{array}$ & $\begin{array}{l}; 3 n=262 \text { for } \mathrm{s} \\
\text { eraction and edt } \\
\text { gr stereotype pel } \\
\text { gma consciousn } \\
23 \text { to } 91 \text {, with scc } \\
\text { to children; } 8 \mathrm{Ag} \\
\text { to being childles } \\
\text { t a slight degree }\end{array}$ & $\begin{array}{l}\text { reotype perc } \\
\text { ation; } 4 \mathrm{n}=2 \\
\text { eption, } 93 \text { fol } \\
\text { ss, } 153 \text { for ex } \\
\text { es below } 52 \\
\text { regate index } \\
9 \text { Ordinal sc } \\
\text { f exclusion } k\end{array}$ & $\begin{array}{l}\text { stigi } \\
\text { clusi } \\
\text { indice } \\
\text { from } \\
\text { ale } f r\end{array}$ & $\begin{array}{l}\text { na } \\
\text { on } \\
\text { ting } \\
10 \text { to } \\
\text { om } 1 \\
\text { se }\end{array}$ \\
\hline
\end{tabular}




\section{Discussion}

This exploratory study is the first to describe the social connection and exclusion from multiple domains of life in Australian society, of women with no children during midlife. The study did not seek to infer causal relationships or control for potential confounding factors associated with social exclusion, such as age and sexual orientation, or interactions between the domains of life. However, the findings provide evidence on the social, civic and service domains of life, which are new areas of research for women with no children during midlife; and augment the limited research on the economic domain. This discussion addresses the social connection and exclusion of women with no children, differences between typologies of women with no children, and the strengths and limitations of the research.

\section{Social exclusion of women with no children}

Women in this study reported feeling negatively stereotyped by others because they have no children. This complements earlier findings that other people stereotyped and had negative attitudes about women with no children (Çopur \& Koropeckyj-Cox, 2010; Koropeckyj-Cox \& Pendell, 2007; LaMastro, 2001). However, midlife women in this study were not conscious of being stigmatised due to having no children, in contrast with the authors' previous research with a younger cohort of Australian women in their reproductive years (2016). This inconsistency may be influenced by a number of factors, including the salience of having no children at different stages of life, both from the perspective of women with no children and others who stigmatise them; and the opportunity for women to adapt to having no children over time. In addition, the inconsistency within this study between women feeling stereotyped and not stigmatised may reflect the measurement of a broader range of experiences by the stigma consciousness scale.

In relation to indicators of exclusion, the findings revealed the extent of connection and exclusion varied between and within the domains of life. In the social domain, midlife women with no children had small social networks, moderate levels of social interaction, moderate levels of social and leisure participation, high levels of social support, and high levels of satisfaction with social support. The findings are consistent with earlier research in which women with no children in their reproductive years reported moderate levels of social interaction and high levels of social support (Turnbull, Graham \& Taket, 2016). This consistency suggests levels of connection and exclusion in the social domain remain stable as women progress through their reproductive years into midlife.

In the civic domain, midlife women had moderate levels of participation in community groups, activities and events, were satisfied with feeling part of the community, and were satisfied with their opportunities to have a say on important issues. Over half $(51.4 \%)$ of the women volunteered in their communities. Interestingly, midlife women in this study reported moderate levels of community participation, compared to research in which women in their reproductive years with no children reported low levels of community participation (Turnbull, Graham \& Taket, 2016). This study's findings are more congruent with research about women during later life, which has found, of Australian women aged 73 to 78 years, unmarried women with no children were more likely than other women to be involved in volunteering (Cwikel et al., 2006); and no reduced likelihood of 'late-life' adults with no children participating in voluntary work or associations, when compared to late-life adults with children (Wenger et al., 2007). As such, the findings may reflect an increasing capacity or tendency of adults to participate in voluntary and community activities as they grow older. 
Midlife women in this study reported a low degree of problems accessing and using services within the service domain, complementing the findings of research on Australian women with no children in their reproductive years (Turnbull, Graham \& Taket, 2016). However, it is possible that aggregating the score for a range of services disguised problems accessing and using particular services.

In the economic domain, the majority of midlife women were employed or selfemployed, and in the upper-middle or high-income brackets, which augments the small body of Australian and international research on women with no children during midlife (Huber et al., 2010; Parr, 2005). In addition, these findings are consistent with research on Australian women with no children in their reproductive years (Turnbull, Graham \& Taket, 2016), suggesting high income and employment rates as indicators of connection in the economic domain remain relatively stable from women's reproductive years into midlife. In relation to the quality of their working lives, women in this study experienced some work-life conflict, which is congruent with other findings that women with no children during their working years experienced work-life conflict and low control over work hours (McNamee \& James, 2012; Swanberg et al., 2005).

The authors have previously suggested (2016) the failure to consistently find exclusion from or connection within any domain of life, may indicate poor quality resources and participation is more pertinent to women with no children than the extent of resources and participation. For women during midlife, this holds true for the economic domain, in which women reported high levels of resources (personal income) and participation (employment), but poor quality working lives in the form of work-life conflict. Conversely, in the social, civic and service domains, midlife women reported low to moderate levels of resources (social networks and social support) and participation (social interaction, social and leisure participation, and community participation); but higher quality resources and participation (satisfaction with social support, satisfaction with feeling part of their communities, satisfaction with opportunities to have a say, and a low degree of problems accessing and using services). As such, for women in midlife, it may be that high quality experiences within some domains ameliorate low levels of resources and participation, while high levels of resources and participation in other domains are tainted by poor quality experiences.

When considering midlife women's self-reported feelings of exclusion from domains of life because they have no children, midlife women perceived more exclusion in the social and the civic domains than the service and economic domains. These results are again consistent with the findings from research with Australian women in their reproductive years with no children (Turnbull, Graham \& Taket, 2016). In the earlier mixed-methods study, triangulation of quantitative and qualitative evidence suggested the variation in perceived exclusion in the different domains may have reflected the greater salience of pronatalist stereotyping and stigmatisation in relation to interpersonal experiences in the social and civic domains, and conversely the greater freedom to participate and accumulate resources in the economic and service domains. The findings from the quantitative component alone of the present study do not enable a determination of whether the same argument can be applied to midlife women with no children. It should also be noted the degree of women's selfreported feelings of exclusion because they have no children is not entirely consistent with the extent of connection and exclusion in all domains. For example, in the social domain, although midlife women reported high levels of social support and satisfaction with social support, almost half $(46.8 \%)$ of the participants reported feeling excluded from social support because they have no children. On the other 
hand, midlife women reported only moderate levels of social interaction with family and friends, and moderate levels of participation in social and leisure activities. These findings were more consistent with the percentages of midlife women who reported feeling excluded from social interaction (46.9\%) and social and leisure participation $(46.8 \%)$ because they have no children. In the economic domain, the low percentages of midlife women who reported feeling excluded because they have no children from material resources $(18.8 \%)$ and employment $(15.1 \%)$ are more reflective of women's high income and employment rates than the impact of work-life conflict on the quality of women's working lives. These findings reveal the complexity of women's experiences, and suggest the more 'objective' measurements of indicators of connection and exclusion may not always reflect women's subjective experiences of feeling excluded because they have no children. Furthermore, it is important to recognise that women's feelings of exclusion because they have no children are self-reported, and accordingly do not establish that having no children causes exclusion. However, self-reported feelings are an important descriptor of midlife women's experiences of having no children.

\section{Differences in social exclusion of typologies of women with no children}

In respect to the indicators of exclusion, few differences between typologies of women with no children reached statistical significance. Circumstantially childless midlife women reported lower levels of social support than voluntarily childless midlife women. This finding is largely consistent with research with women in their reproductive years (Turnbull, Graham \& Taket, 2016), in which circumstantially childless women had lower levels of social support than voluntarily and involuntarily childless women. In this study, circumstantially childless midlife women also reported a greater degree of problems accessing and using services than voluntarily childless midlife women. A similar difference did not emerge from the authors' research with women in their reproductive years (2016). However, given that circumstantially childless midlife women in this study were more likely than involuntarily and voluntarily childless midlife women to be single, and constituted the majority of women who identified as lesbian or bisexual, it is important acknowledge that partner status and sexual orientation may have confounded these findings. A further influence on these results may be the kinds of services accessed by different typologies of women with no children.

The absence of statistically significant differences in the civic and economic domains was consistent with women in their reproductive years (2016). The lack of differences in employment rates and income levels contrast with the findings of United States and French studies, in which voluntarily childless women's incomes and workforce participation rates were greater than those of other women with no children (Abma \& Martinez, 2006; Waren \& Pals, 2013). Those studies related to women in their reproductive years, rather than women in midlife.

There were also no differences in participants self-reported degree of feeling excluded from the domains of life because they have no children. The differences that approached significance at a conservative level of 0.01 , suggested involuntarily childless midlife women felt more excluded from social support and social interaction than voluntarily childless midlife women. The findings vary substantially from earlier research with women during their reproductive years (Turnbull, Graham \& Taket, 2016), in which circumstantially childless women felt more excluded from social interaction and social support than voluntarily childless women, and involuntarily and circumstantially childless women felt more excluded from community life than voluntarily childless women. As previously posited, the incongruences between the life stages, with fewer differences between typologies of women with no children during midlife than their reproductive years, may be influenced by the salience of 
having no children at different stages of life and the capacity to adapt over time to having no children. Furthermore, the authors' mixed-methods research with women in their reproductive years (2016) suggested women's different circumstances and reasons for having no children may have influenced their nuanced experiences of connection and exclusion. The presence of fewer differences between involuntarily, circumstantially and voluntarily childless women during midlife suggests such differences may become less influential over time. In particular, whether having no children was temporary or permanent would become increasingly irrelevant, as the majority of women during midlife would identify as permanently having no children.

\section{Strengths, limitations and future directions}

The research had a number of limitations. The cross-sectional design prevented any statistical investigation of causality. The recruitment strategy, which relied substantially on social media and electronic communications, may not have been the most appropriate approach for this age group, suggesting the need for additional recruitment channels. In addition, the 55 per cent completion rate may have introduced selection bias; self-selection due to the absence of a sampling frame may have resulted in a sample more likely to experience exclusion; and non-probability sampling limited external validity. The small sizes of some sub-groups, particularly involuntarily childless women, limit the ability to find statistically significant differences between typologies of childless women. Furthermore, while a conservative significance level of 0.01 was used, it is possible some statistically significant findings occurred by chance. Finally, due to its exploratory nature, the study did not control for confounding factors or potential interactions between the domains of life, meaning there may have been explanations other than having no children for the extent of connection and exclusion, such as partner status, sexual orientation and age. Instead, the study focused on describing the social exclusion across and within the domains of life among a sample of midlife women with no children.

In spite of its limitations, this study was the first to describe the social connection and exclusion in multiple domains of life in Australian society, of women with no children during midlife. Furthermore, measuring a broad range of indicators enabled a detailed description of the complex phenomenon of social exclusion. Where available, using existing and validated scales minimised measurement bias; while self-administration reduced the likelihood of socially desirable responses.

Finally, although the sample's demographic characteristics diverged from comparable Australian data, participants' high rates of educational attainment, employment, personal income and voluntary childlessness were congruent with existing research on the socio-demographic characteristics of women with no children (Abma \& Martinez, 2006; Miranti et al., 2009; Waren \& Pals, 2013).

This discussion points to a number of directions for future research, in order to elucidate the similarities to and differences from research with women during midlife and their reproductive years, and possible interactions between indicators of exclusion and socio-demographic characteristics. Future detailed analyses of each domain of life will draw on data from women during their reproductive years and midlife, enabling analyses of interaction effects between intersecting characteristics such as age, partner status and sexual orientation, and potential interactions between the domains of life.

\section{Conclusion}

This research has found that midlife women with no children experience complex patterns of connection and exclusion in different domains of life in Australian society, 
with the extent and quality of connection and exclusion varying within and between the domains of life. Midlife women report feeling more excluded because they have no children in the social and civic domains than the service and economic domains. The research further suggests there are few differences in the extent of stereotyping, stigmatisation, connection and exclusion of involuntarily, circumstantially and voluntarily childless women during midlife. Social exclusion is a key social determinant of health and wellbeing, reinforcing the importance of ensuring all women have opportunities for connection in all domains of life, regardless of their motherhood status. We infer this entails challenging pronatalist ideologies, discourses, policies, cultures, beliefs, attitudes and behaviours at all levels of society. 


\section{References}

Abma, J. C., \& Martinez, G. M. (2006). Childlessness among older women in the United States: Trends and profiles. Journal of Marriage and Family, 68(4), 1045-1056.

Albertini, M., \& Mencarini, L. (2014). Childlessness and support networks in later life: New pressures on familistic welfare states? Journal of Family Issues, 35(3), 331-357.

Australian Bureau of Statistics. (2007). 2006 Census community profile series - Basic community profile: Australia. Retrieved from http://www.censusdata.abs.gov.au/census services/getproduct/census/2006/ communityprofile/0?opendocument\&navpos $=220$

Australian Bureau of Statistics. (2008). Australian social trends, 2008. Retrieved from http://www.abs.gov.au/AUSSTATS/abs@.nsf/Lookup/4102.0Chapter3202008

Australian Bureau of Statistics. (2010). General social survey. Retrieved from http://www.abs.gov.au/AUSSTATS/abs@.nsf/DetailsPage/4159.0.55.0022010 ?OpenDocument

Australian Bureau of Statistics. (2011). Census household form. Retrieved from http://abs.gov.au/ausstats/abs@.nsf/Lookup/2901.0MainFeatures802011/\$FI LE/2011 Census Household Form.pdf

Australian Bureau of Statistics. (2012a). 2011 Census of population and housing Basic community profile: Australia. Retrieved from http://www.censusdata.abs.gov.au/census services/getproduct/census/2011/ communityprofile/0?opendocument\&navpos $=220$

Australian Bureau of Statistics. (2012b). Income data in the census. Retrieved from http://www.abs.gov.au/websitedbs/censushome.nsf/home/factsheetsuid?open document\&navpos $=450$

Australian Bureau of Statistics. (2013). Australian health survey: Users' guide, 201113. Retrieved from http://www.abs.gov.au/AUSSTATS/abs@.nsf/Lookup/4363.0.55.001Glossary 12011-13?OpenDocument

Baumle, A. K. (2009). The cost of parenthood: Unravelling the effects of sexual orientation and gender on income. Social Science Quarterly, 90(4), 983-1002.

Berdahl, J. L., \& Moon, S. H. (2013). Workplace mistreatment of middle class workers based on sex, parenthood, and caregiving. Journal of Social Issues, 69(2), 341-366.

Bernstein, A. B. (2001). Motherhood, health status, and health care. Women's Health Issues, 11(3), 173-184.

Butler, J. (2011). Gender trouble: Feminisim and the subversion of identity. New York: Routledge.

Cannold, L. (2005). What, no baby? Why women are losing the freedom to mother, and how they can get it back. Fremantle: Curtin University Books.

Carey, G., Graham, M., Shelley, J., \& Taket, A. (2009). Discourse, power and exclusion: The experiences of childless women. In A. Taket, B. Crisp, A. Nevill, G. Lamaro, M. Graham, \& S. Barter-Godfrey (Eds.), Theorising social exclusion. London: Routledge.

Casper, W. J., Weltman, D., \& Kwesiga, E. (2007). Beyond family-friendly: The construct and measurement of singles-friendly work culture. Journal of Vocational Behavior, 70(3), 478-501.

Collins, P.H., (2000). Black feminist thought: knowledge, consciousness and the politics of empowerment ( $2^{\text {nd }}$ ed. $)$. New York: Routledge.

Çopur, Z., \& Koropeckyj-Cox, T. (2010). University students' perceptions of childless couples and parents in Ankara, Turkey. Journal of Family Issues, 31(11), 1481-1506. 
Cummins, R. A., Eckersley, R., Pallant, J., Van Vugt, J., \& Misajon, R. (2003). Developing a national index of subjective wellbeing: The Australian Unity Wellbeing Index. Social Indicators Research, 64(2), 159-190.

Cwikel, J., Gramotnev, H., \& Lee, C. (2006). Never-married childless women in Australia: Health and social circumstances in older age. Social Science and Medicine, 62(8), 1991-2001.

Daniluk, J. C. (2001). Reconstructing their lives: a longitudinal, qualitative analysis of the transition to biological childlessness for infertile couples. Journal of Counseling \& Development, 79(4), 439-449.

Dermott, E., Fahmy, E., Gordon, D., Heslop, P., Levitas, R., \& Patsios, D. (2012). Poverty and social exclusion in the UK: Living standards questionnaire 2012. Bristol: PSE: UK.

Dykstra, P. A., \& Wagner, M. (2007). Pathways to childlessness and late-life outcomes. Journal of Family Issues, 28(11), 1487-1517.

George, L. K., Blazer, D. G., Hughes, D. C., \& Fowler, N. (1989). Social support and the outcome of major depression. The British Journal of Psychiatry, 154(4), 478-485.

Gillespie, R. (2000). When no means no: Disbelief, disregard and deviance as discourses of voluntary childlessness. Women's Studies International Forum, 23(2), 223-234.

Graham, M., \& Rich, S. (2012a). Representations of childless women in the Australian print media. Feminist Media Studies, 14(3), 500-518.

Graham, M., \& Rich, S. (2012b). What's 'childless' got to do with it? Geelong: Alfred Deakin Research Institute.

Grundy, E., \& Read, S. (2012). Social contacts and receipt of help among older people in England: Are there benefits of having more children? The Journals of Gerontology Series B: Psychological Sciences and Social Sciences, 67(6), 742-754.

Hamilton, E. A., Gordon, J. R., \& Whelan-Berry, K. S. (2006). Understanding the work-life conflict of never-married women without children. Women in Management Review, 21(5), 393-415.

Hank, K., \& Wagner, M. (2013). Parenthood, marital status, and well-being in later life: Evidence from SHARE. Social Indicators Research, 1-15.

Heard, G. (2006). Pronatalism under Howard. People and Place, 14(3), 12-25.

Huber, S., Bookstein, F. L., \& Fieder, M. (2010). Socioeconomic status, education, and reproduction in modern women: an evolutionary perspective. American Journal of Human Biology, 22(5), 578-587.

Hughes, D., Blazer, D., \& Hybels, C. (1990). Duke social support index (DSSI): A working paper (revised). Durham, N.C.: Duke University Medical Center.

Kopper, B. A., \& Smith, M. S. (2001). Knowledge and attitudes toward infertility and childless couples. Journal of Applied Social Psychology, 31(11), 2275-2291.

Koropeckyj-Cox, T., \& Call, V. R. (2007). Characteristics of older childless persons and parents: Cross-national comparisons. Journal of Family Issues, 28(10), $1362-1414$.

Koropeckyj-Cox, T., \& Pendell, G. (2007). Attitudes about childlessness in the United States: Correlates of positive, neutral, and negative responses. Journal of Family Issues, 28(8), 1054-1082.

Koropeckyj-Cox, T. (2002). Beyond parental status: Psychological well being in middle and old age. Journal of Marriage and Family, 64(4), 957-971.

LaMastro, V. (2001). Childless by choice? Attributions and attitudes concerning family size. Social Behavior and Personality: an international journal, 29(3), 231-243.

Lampman, C., \& Dowling-Guyer, S. (1995). Attitudes toward voluntary and involuntary childlessness. Basic and Applied Social Psychology, 17(1-2), 213222. 
Levitas, R. (2006). The concept and measurement of social exclusion. In C. Pantazis, D. Gordon, \& R. Levitas (Eds.), Poverty and social exclusion in Britain: the millennium survey (pp. 123-160). Bristol: Policy Press.

Levitas, R., Pantazis, C., Fahmy, E., Gordon, D., Lloyd, E., \& Patsios, D. (2007). The multi-dimensional analysis of social exclusion. London: Cabinet Office.

McNamee, B. G., \& James, G. D. (2012). The impact of child-rearing status on perceptual and behavioural predictors of ambulatory blood pressure variation among working women. Annals of Human Biology, 39(6), 490-498.

Melbourne Institute of Applied Economic and Social Research. (2012). Household, income and labour dynamics in Australia (HILDA) survey: Wave 12M self completion questionnaire. Retrieved from https://www.melbourneinstitute.com/hilda/doc/questionnaires/q12.html

Miranti, R., McNamara, J., Tanton, R., \& Yap, M. (2009). A narrowing gap? Trends in the childlessness of professional women in Australia 1986-2006. Journal of Population Research, 26(4), 359-379.

Misra, J., Budig, M. J., \& Boeckmann, I. (2011). Cross-national patterns in individual and household employment and work hours by gender and parenthood. Research in the Sociology of Work, 22, 169-207.

Mumtaz, Z., Shahid, U., \& Levay, A. (2013). Understanding the impact of gendered roles on the experiences of infertility amongst men and women in Punjab. Reproductive Health, 10(1), 3-12.

Parr, N. J. (2005). Family background, schooling and childlessness in Australia. Journal of Biosocial Science, 37(2), 229-243.

Pinel, E. C. (1999). Stigma consciousness: the psychological legacy of social stereotypes. Journal of Personality and Social Psychology, 76(1), 114-128.

Popay, J., Escorel, S., Hernández, M., Johnston, H., Mathieson, J., \& Rispel, L. (2008). Understanding and tackling social exclusion: Final report to the WHO Commission on Social Determinants of Health from the Social Exclusion Knowledge Network. Lancaster, UK: WHO Social Exclusion Knowledge Network.

Pope, J., \& Zhang, W. (2010). Indicators of community strength at the local government area level in Victoria 2008. Melbourne: Victorian Department of Planning and Community Development.

Rich, S., Taket, A., Graham, M., \& Shelley, J. (2011). 'Unnatural', 'unwomanly', 'uncreditable' and 'undervalued': The significance of being a childless woman in Australian society. Gender Issues, 28(4), 226-247.

Sawer, M. (2013). Misogyny and misrepresentation women in Australian parliaments. Political Science, 65(1), 105-117.

Sherbourne, C. D., \& Stewart, A. L. (1991). The MOS social support survey. Social Science and Medicine, 32(6), 705-714.

Smith, A., Rissel, C. E., Richters, J., Grulich, A. E., \& Visser, R. O. (2003). Sex in Australia: sexual identity, sexual attraction and sexual experience among a representative sample of adults. Australian and New Zealand Journal of Public Health, 27(2), 138-145.

Somers, M. D. (1993). A comparison of voluntarily childfree adults and parents. Journal of Marriage and the Family, 55(3), 643-650.

Swanberg, J. E., Pitt-Catsouphes, M., \& Drescher-Burke, K. (2005). A question of justice: Disparities in employees' access to flexible schedule arrangements. Journal of Family Issues, 26(6), 866-895.

Turnbull, B., Graham, M., \& Taket, A. (2016). Social exclusion of Australian childless women in their reproductive years. Social Inclusion, 4(1), 102-115.

Veevers, J. E. (1979). Voluntary childlessness. Marriage \& Family Review, 2(2), 126. 
Vikström, J., Bladh, M., Hammar, M., Marcusson, J., Wressle, E., \& Sydsjö, G. (2011). The influences of childlessness on the psychological well-being and social network of the oldest old. BMC Geriatrics, 11(1), 78.

Wagner, J., Wrzus, C., Neyer, F. J., \& Lang, F. R. (2015). Social network characteristics of early midlife voluntarily and involuntarily childless couples. Journal of Family Issues, 36(1), 87-110.

Waren, W., \& Pals, H. (2013). Comparing characteristics of voluntarily childless men and women. Journal of Population Research, 30(2), 151-170.

Waumsley, J. A., Houston, D. M., \& Marks, G. (2010). What about us? Measuring the work-life balance of people who do not have children. Review of European Studies, 2(2), 3-17.

Wenger, G. C., Dykstra, P. A., Melkas, T., \& Knipscheer, K. C. (2007). Social embeddedness and late-life parenthood: Community activity, close ties, and support networks. Journal of Family Issues, 28(11), 1419-1456.

Women's Health Australia. (2010). Australian longitudinal study on women's health: Sixth survey for the women of the 1946-51 cohort 2010. Retrieved from https://www.ada.edu.au/ADAData/questionnaires/ADA.QUESTIONNAIRE.01 017-mid6.pdf 


\section{Biographical Notes}

Beth Turnbull is a PhD candidate in the School of Health and Social Development, Deakin University. Her research seeks to understand the social connection and exclusion in different areas of life of women with no children, and how those experiences influence health and wellbeing.

Dr Melissa Graham is a Senior Lecturer in the School of Health and Social Development, Deakin University. She is the Deputy Director of the Centre for Health through Action on Social Exclusion (CHASE). Dr Graham's research explores the lives of women without children with a particular focus on social exclusion, and health and wellbeing.

Ann Taket holds the chair in Health and Social Exclusion within the School of Health and Social Development, and is Director of the Centre for Health through Action on Social Exclusion (CHASE) at Deakin University. Professor Taket has over thirty years' experience in public health related research, with particular interests in research directed at understanding the complex interactions between social exclusion and health, prevention and intervention in violence and abuse and the value of human rights based approaches in policy and practice. 